BRAVZULIAN JOURNAL

OF MEDICAL AND BIOLOGICAL RESFARCH

www.bjournal.com.br
ISSN 0100-879X

Volume 43 (5) 381-496 May 2011

BIOMEDICAL SCIENCES

AND

CLINICAL INVESTIGATION

Braz J Med Biol Res, May 2011, Volume 44(5) 469-476

doi: 10.1590/S0100-879X2011007500034

Rosuvastatin prevents myocardial necrosis in an experimental model of acute myocardial infarction

P.M.M. Dourado, J.M. Tsutsui, M.B.P. Landim, A. Casella Filho, T.F.G. Galvao, V.D. Aiello, W. Mathias Jr., P.L. da Luz and A.C.P. Chagas

The Brazilian Journal of Medical and Biological Research is partially financed by

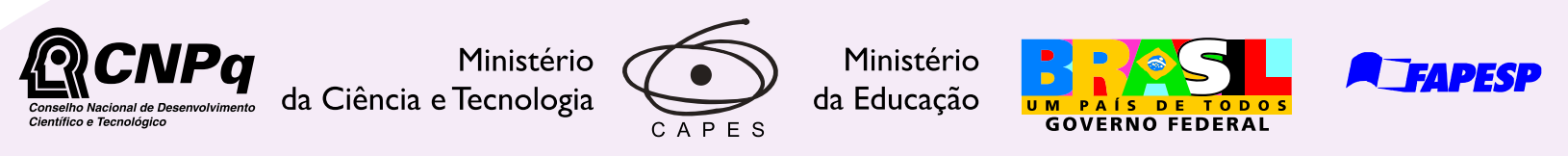

Institutional Sponsors
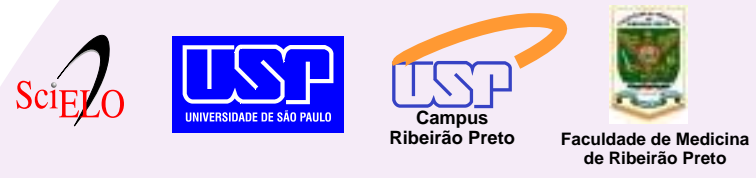
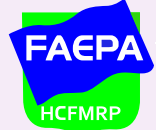

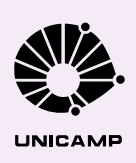

Ф SHIMADZU H

GE Healthcare
Hotsite of proteomics metabolomics developped by:

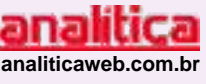

Thermo SCIENTIFIC 


\title{
Rosuvastatin prevents myocardial necrosis in an experimental model of acute myocardial infarction
}

\author{
P.M.M. Dourado ${ }^{1}$, J.M. Tsutsui ${ }^{2}$, M.B.P. Landim ${ }^{1}$, A. Casella Filho ${ }^{1}$, T.F.G. Galvao ${ }^{1}$, \\ V.D. Aiello ${ }^{3}$, W. Mathias Jr. ${ }^{2}$, P.L. da Luz ${ }^{1}$ and A.C.P. Chagas ${ }^{1}$ \\ 1 Unidade Clínica de Aterosclerose, ${ }^{2}$ Serviço de Ecocardiografia, ${ }^{3}$ Serviço de Patologia, Instituto do Coração, \\ Hospital das Clínicas, Faculdade de Medicina, Universidade de São Paulo, São Paulo, SP, Brasil
}

\begin{abstract}
Dyslipidemia is related to the progression of atherosclerosis and is an important risk factor for acute coronary syndromes. Our objective was to determine the effect of rosuvastatin on myocardial necrosis in an experimental model of acute myocardial infarction (AMI). Male Wistar rats (8-10 weeks old, 250-350 g) were subjected to definitive occlusion of the left anterior descending coronary artery to cause AMI. Animals were divided into 6 groups of 8 to 11 rats per group: G1, normocholesterolemic diet; G2, normocholesterolemic diet and rosuvastatin $\left(1 \mathrm{mg} \cdot \mathrm{kg}^{-1} \cdot\right.$ day $\left.^{-1}\right) 30$ days after AMl; G3, normocholesterolemic diet and rosuvastatin ( $\left.1 \mathrm{mg} \cdot \mathrm{kg}^{-1} \cdot \mathrm{day}^{-1}\right) 30$ days before and after AMI; G4, hypercholesterolemic diet; G5, hypercholesterolemic diet and rosuvastatin ( $\left.1 \mathrm{mg} \cdot \mathrm{kg}^{-1} \cdot \mathrm{day}^{-1}\right) 30$ days after AMl; G6, hypercholesterolemic diet and rosuvastatin $\left(1 \mathrm{mg} \cdot \mathrm{kg}^{-1} \cdot \mathrm{day}^{-1}\right) 30$ days before and after AMI. Left ventricular function was determined by echocardiography and percent infarct area by histology. Fractional shortening of the left ventricle was normal at baseline and decreased significantly after AMI $(P<0.05$ in all groups), being lower in G4 and G5 than in the other groups. No significant difference in fractional shortening was observed between G6 and the groups on the normocholesterolemic diet. Percent infarct area was significantly higher in G4 than in G3. No significant differences were observed in infarct area among the other groups. We conclude that a hypercholesterolemic diet resulted in reduced cardiac function after AMI, which was reversed with rosuvastatin when started 30 days before AMI. A normocholesterolemic diet associated with rosuvastatin before and after AMI prevented myocardial necrosis when compared with the hypercholesterolemic condition.
\end{abstract}

Key words: Acute myocardial infarction; Rosuvastatin; Hypercholesterolemia; Myocardial necrosis

\section{Introduction}

Dyslipidemia has been known to result in the progression of atherosclerosis and in an increased incidence of thrombotic complications in patients with coronary artery disease (CAD) (1). Many studies have shown that treatment of dyslipidemias is beneficial in decreasing the number of coronary events. However, the role of statins in myocardial protection after acute myocardial infarction (AMI) is not known. Experimental $(2,3)$ and clinical studies $(4,5)$ have demonstrated that coronary reperfusion in the setting of AMI reduces the extent of final necrosis and improves ventricular function, favorably influencing the late survival of infarcted patients (6). These effects seem to be independent of the efficiency of intervention (7).

The statins, pharmacologically denominated as inhibi- tors of 3-hydroxy-3-methylglutaryl coenzyme A(HMG-CoA) reductase, were developed in the 1970's (8). Since then, new chemical structures of statins have been developed and have been widely used for the treatment of hypercholesterolemia, reducing the levels of low-density lipoprotein cholesterol (LDL-C) and triglycerides (9). Some recently published studies have demonstrated that rosuvastatin is effective in reducing LDL-C levels and in increasing high-density lipoprotein cholesterol (HDL-C) $(10,11)$, is safe in elderly patients with systolic cardiac dysfunction, and reduces the risk of cardiovascular events in healthy patients without dyslipidemia, but with high levels of high sensitivity C-reactive protein (12). Statins are also known to have pleiotropic effects, which seem to be independent

Correspondence: P.M.M. Dourado, Unidade Clínica de Aterosclerose, InCor, HC, FM, USP, Av. Dr. Enéas Carvalho Aguiar, 44, 05403-900 São Paulo, SP, Brasil. Fax: +55-16-3069-5000. E-mail: pmdourado@terra.com.br

Received November 5, 2010. Accepted March 10, 2011. Available online March 25, 2011. Published May 16, 2011. 
of their lipid-lowering action, such as anti-atherosclerotic, anti-inflammatory, anti-thrombosis, and anti-oxidant effects. These properties could potentially reduce the necrotic area in the setting of AMI. However, the effects of rosuvastatin on myocardial necrosis have not been clearly demonstrated in the literature.

Left ventricular dysfunction is the most frequent consequence of CAD and AMI. AMI frequently results in ventricular remodeling with dilation of the left ventricular cavity and wall thinning caused by mural stress (13). The development of heart failure after AMI is mainly caused by apoptosis and structural changes in myocardial tissue in reaction to metalloproteinase (MMP) enzymes $(14,15)$. Moreover, pathological degradation of collagen after several episodes of myocardial ischemia was associated with increased inflammatory activity in tissues and increased levels of angiotensin II and aldosterone, which are responsible for ventricular remodeling $(16,17)$.

The aim of this study was to evaluate the role of rosuvastatin on myocardial necrosis and cardiac function within the setting of experimental model of AMI.

\section{Material and Methods}

The study was approved by the Institutional Review Board and performed in the Myocardial Ischemia Investigation Laboratory of the Heart Institute (InCor), University of São Paulo Medical School. The protocol was in accordance with the position of the American Heart Association Guidelines for Animal Research Use (18).

We studied adult male Wistar rats weighing 250 to $350 \mathrm{~g}$, aged 8 to 10 weeks. Animals were maintained at $22-24^{\circ} \mathrm{C}, 5$ animals per cage, with free access to water. Animals assigned to the normocholesterolemic diet received standard chow (Nuvital ${ }^{\circledR}$ from Nuvilab Nutrientes

Table 1. Composition of the hypercholesterolemic diet used in the intervention group.

\begin{tabular}{lc}
\hline Component & Quantity (\%) \\
\hline Carbohydrates* $^{*}$ & 51.95 \\
Protein $^{\dagger}$ & 20.00 \\
Lipids $^{\ddagger}$ & 18.00 \\
Fibers & 5.00 \\
Mineral mix & 1.00 \\
Vitamin mix & 3.00 \\
L-cystine & 0.30 \\
Choline bitartrate & 0.25 \\
Cholic acid & 0.50 \\
\hline
\end{tabular}

${ }^{*}$ Carbohydrate fractions: $55.8 \%$ starch, $25.8 \%$ maltodextrin and $18.4 \%$ sucrose. TProtein: commercial casein ( $85 \%$ protein). łLipid fraction: $66.7 \%$ coconut oil, $26.4 \%$ soybean oil and $6.9 \%$ cholesterol. Vitamin and mineral mixtures were based on the recommendations of the American Institute of Nutrition (AIN-93G).
S/A, Brazil) while those assigned to the hypercholesterolemic diet received a special diet containing colic acid (19) (Table 1). Our goal with this procedure was to induce hypercholesterolemia in rats with a normal genetic background. All rats underwent a surgical procedure for definitive occlusion of the left anterior descending (LAD) coronary artery to cause AMI and were assigned to 6 groups of 8-11 rats per group: G1, normocholesterolemic diet without rosuvastatin; G2, normocholesterolemic diet and rosuvastatin ( $1 \mathrm{mg} \cdot \mathrm{kg}^{-1} \cdot$ day $\left.^{-1}\right) 30$ days after AMI; G3, normocholesterolemic diet and rosuvastatin $\left(1 \mathrm{mg} \mathrm{kg}^{-1} \cdot \mathrm{day}^{-1}\right)$ 30 days before and 30 days after AMI; G4, hypercholesterolemic diet 30 days before and after AMI without rosuvastatin; G5, hypercholesterolemic diet 30 days before and after AMI and rosuvastatin $\left(1 \mathrm{mg} \cdot \mathrm{kg}^{-1} \cdot \mathrm{day}^{-1}\right) 30$ days after AMI; G6, hypercholesterolemic diet 30 days before and 30 days after $\mathrm{AMI}$ and rosuvastatin $\left(1 \mathrm{mg} \cdot \mathrm{kg}^{-1} \cdot \mathrm{day}^{-1}\right)$ 30 days before and 30 days after AMI.

The experimental protocol for each group is illustrated in Figure 1.

\section{Surgical procedure}

The animals were anesthetized with intraperitoneal xylazine $(10 \mathrm{mg} / \mathrm{kg})$ and ketamine $(90 \mathrm{mg} / \mathrm{kg})$. All surgical procedures were performed by the same operator, who was blind to animal group. An arterial cannula was inserted into the left ventricle to measure left ventricular end-diastolic pressure before and $5 \mathrm{~min}$ after AMI. The catheter position was confirmed by the characteristic pulse pressure. Left ventricular end-diastolic pressure was recorded beat-to-beat (DataQ Instruments, Inc., USA). The rats were mechanically ventilated (Harvard respirator, $2.5 \mathrm{~mL}, 75$ to 80 strikes $/ \mathrm{min}$ ) and their chest wall was opened with a left thoracotomy. The LAD coronary artery was then permanently occluded with a $5 / 0$-silk thread close to its origin. After AMI, the animals were allowed to heal for 30 days.

\section{Inflammatory markers}

In all groups, blood samples were collected from the right jugular vein before and after LAD occlusion. Serum levels of tumor necrosis factor alpha (TNF- $\alpha)$, interleukin 6 (IL-6), metalloproteinase 2 (MMP-2), and asymmetric dimethylarginine (ADMA) were determined by ELISA.

\section{Echocardiography}

Echocardiography was performed using a commercially available platform (HDI 5000, Philips Medical Systems, USA) equipped with a 12-MHz transducer. Echocardiographic imaging was adjusted to optimize image acquisition and echocardiographic parameters were then held constant for each experiment. The images were acquired at baseline, before LAD occlusion, and at 30 days after AMI. Left ventricular end-diastolic and end-systolic diameters were obtained by unidimensional 

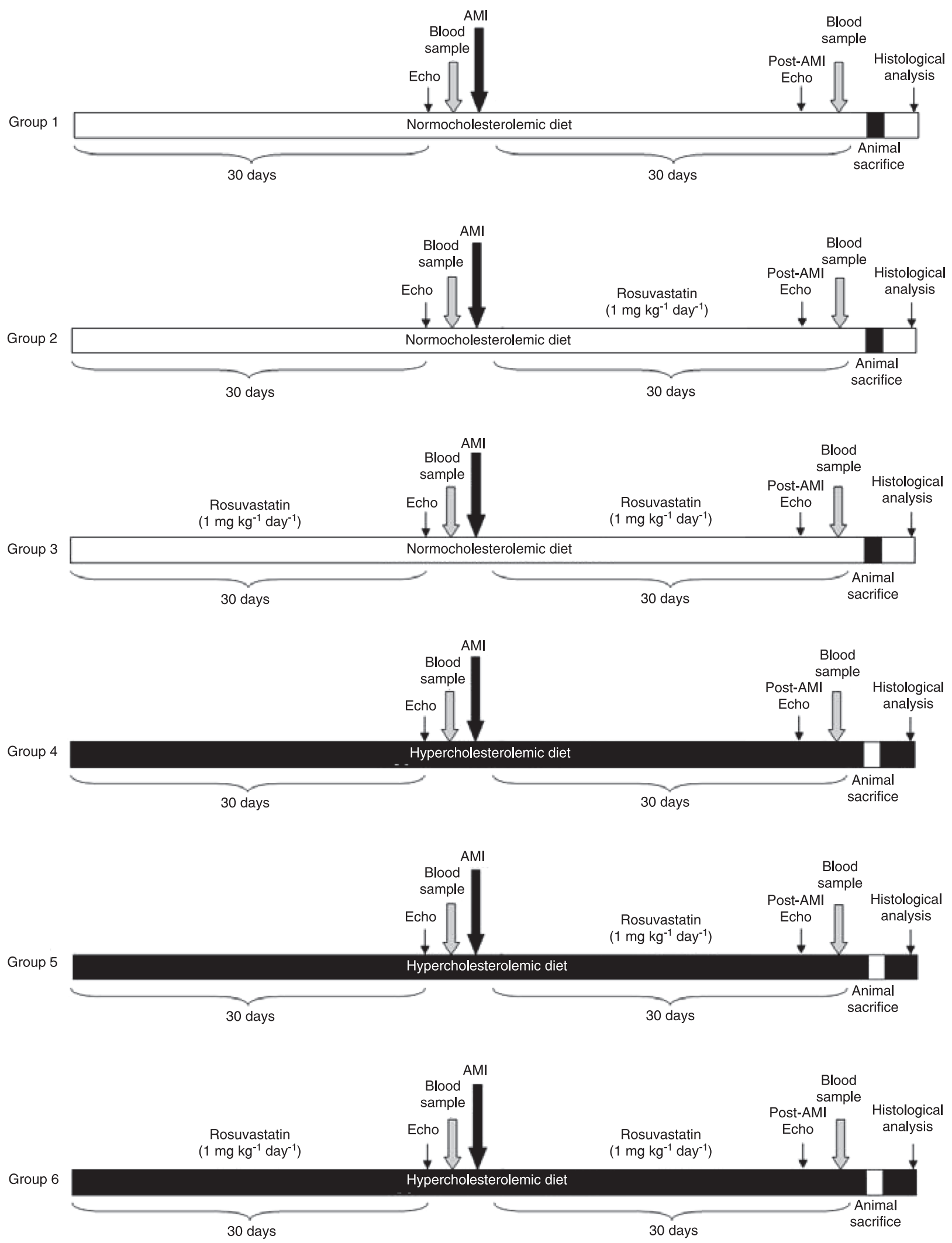

Figure 1. Experimental protocol. $\mathrm{AMI}=$ acute myocardial infarction. 
mode at the level of papillary muscles. Fractional shortening was determined as the difference of end-diastolic and end-systolic diameters divided by end-diastolic diameter. Left ventricular volumes were determined by the biplane method of disks (Simpson's rule). The myocardial thickness of the interventricular septum and inferoposterior wall of the left ventricle was measured and left ventricular mass was corrected by each animal's body weight. Echocardiographic measurements were performed on all rats by the same investigator, who was blind to the animal groups.

\section{Histology}

After 30 days of $A M I$, rats were anesthetized with intraperitoneal xylazine $(10 \mathrm{mg} / \mathrm{kg})$ and ketamine $(90$ $\mathrm{mg} / \mathrm{kg}$ ), and the left ventricle was cannulated with a needle for retrograde perfusion.

The animals' heart was arrested in diastole by perfusion with $0.9 \% \mathrm{NaCl}$ plus $14 \mathrm{mM} \mathrm{KCl}$ solution (pressure equal to a 13-cm water column), followed by buffered formalin for tissue fixation. Excised hearts were immersed in formalin for $24 \mathrm{~h}$. The heart was then weighed on a precision scale (Mettler-Toledo AB104, Mettler-Toledo, USA). A median slice of the heart sectioned at the level of the papillary muscles was processed for microscopic analysis. Sections of $3 \mu \mathrm{m}$ were stained with hematoxylin-eosin for qualitative assessment, and with Masson's trichrome (blue stain) for quantification of the scar area. The left ventricular

Table 2. Characteristics of the animals in each treatment group.

\begin{tabular}{lcccccc}
\hline & $\mathrm{G} 1(\mathrm{~N}=11)$ & $\mathrm{G} 2(\mathrm{~N}=8)$ & $\mathrm{G} 3(\mathrm{~N}=9)$ & $\mathrm{G} 4(\mathrm{~N}=10)$ & $\mathrm{G} 5(\mathrm{~N}=8)$ & $\mathrm{G} 6(\mathrm{~N}=8)$ \\
\hline Baseline & & & & & & \\
Weight (g) & $250 \pm 22^{*}$ & $250 \pm 40^{*}$ & $261 \pm 33^{*}$ & $342 \pm 31^{*}$ & $372 \pm 25^{*}$ & $366 \pm 18^{*}$ \\
HR (bpm) & $329 \pm 45$ & $310 \pm 47$ & $265 \pm 22$ & $310 \pm 39$ & $323 \pm 33$ & $275 \pm 37$ \\
LVDD (mm) & $5.8 \pm 1.1^{\Delta}$ & $5.2 \pm 0.5^{\Delta}$ & $6.5 \pm 0.7^{\Delta}$ & $6.1 \pm 0.7^{\Delta}$ & $6.3 \pm 0.5^{\Delta}$ & $6.5 \pm 0.3^{\Delta}$ \\
EDV (mL) & $0.42 \pm 0.21^{+}$ & $0.3 \pm 0.05^{+}$ & $0.54 \pm 0.12^{+}$ & $0.53 \pm 0.12^{+}$ & $0.67 \pm 0.09^{+}$ & $0.72 \pm 0.12^{+}$ \\
EF (\%) & $0.81 \pm 0.06$ & $0.81 \pm 0.03$ & $0.79 \pm 0.05$ & $0.83 \pm 0.04$ & $0.78 \pm 0.05$ & $0.75 \pm 0.03$ \\
ILVM (g/kg) & $1.32 \pm 0.16$ & $1.24 \pm 0.19$ & $1.35 \pm 0.15$ & $1.18 \pm 0.20$ & $1.14 \pm 0.12$ & $1.22 \pm 0.11$ \\
30 days after AMI & & & & & & \\
Weight (g) & $356 \pm 33^{*}$ & $332 \pm 42^{*}$ & $331 \pm 39^{*}$ & $433 \pm 44^{*}$ & $436 \pm 55^{*}$ & $436 \pm 54^{*}$ \\
HR (bpm) & $287 \pm 36$ & $300 \pm 27$ & $297 \pm 34$ & $293 \pm 24$ & $293 \pm 24$ & $295 \pm 20$ \\
LVDD (mm) & $8.3 \pm 1.2^{\Delta}$ & $7.7 \pm 0.6^{\Delta}$ & $7.6 \pm 0.6^{\Delta}$ & $8.6 \pm 1.3^{\Delta}$ & $8.6 \pm 1.3^{\Delta}$ & $8.4 \pm 0.9^{\Delta}$ \\
EDV (mL) & $0.92 \pm 0.25^{+}$ & $0.84 \pm 0.14^{+}$ & $0.74 \pm 0.11^{+}$ & $1.15 \pm 0.32^{+}$ & $1.19 \pm 0.28^{+}$ & $1.22 \pm 0.43^{+}$ \\
EF $(\%)$ & $0.46 \pm 0.10$ & $0.46 \pm 0.09$ & $0.44 \pm 0.09$ & $0.38 \pm 0.12$ & $0.38 \pm 0.14$ & $0.41 \pm 0.14$ \\
HW (g) & $1.79 \pm 0.27$ & $1.68 \pm 0.24$ & $1.65 \pm 0.15$ & $1.78 \pm 0.22$ & $1.88 \pm 0.13$ & $1.82 \pm 0.11$ \\
ILVM (g/kg) & $1.71 \pm 0.21$ & $1.61 \pm 0.22$ & $1.76 \pm 0.17$ & $1.67 \pm 0.21$ & $1.66 \pm 0.16$ & $1.59 \pm 0.15$ \\
\hline
\end{tabular}

Data are reported as means \pm SD. See Figure 1 for protocol. $H R=$ heart rate; LVDD = left ventricular diastolic diameter; EDV = end-diastolic volume; $E F=$ left ventricular ejection fraction; ILVM = indexed left ventricular mass; HW = heart weight. ${ }^{*} \mathrm{P}<0.05 \mathrm{G} 1, \mathrm{G} 2$, G3 compared to G4, G5, G6 at baseline and also at 30 days after AMI (ANOVA). ${ }^{\triangle} \mathrm{P}<0.05$ all groups at baseline compared to 30 days after AMI (ANOVA for repeated measures). ${ }^{+} \mathrm{P}<0.05$ all groups at baseline compared to 30 days after AMI (ANOVA for repeated measures). muscle area and interventricular septum thickness were also measured. Histomorphometric analyses were performed by the same person, who was blind to the treatment groups. Only one histological section was examined, and the healed infarction was delineated by computerized microscopy (Leica Imaging Systems, USA) (20) in order to calculate the percent area relative to the total area of the left ventricular myocardium. In fact, since the animals were kept alive for 1 month after infarction, the fibrotic scar was measured.

\section{Statistical analysis}

Quantitative data are reported as means \pm SD. The variables measured in only one condition were evaluated by analysis of variance (ANOVA) with a classification factor, when significant. The Tukey test was applied to determine differences between groups. The variables, measured in more than one condition (pre-and post-AMI), were evaluated by ANOVA for repeated measures. Pre- and post-infarction biochemical levels were compared by the Wilcoxon sign test. Comparisons of all groups were performed using the Kruskal-Wallis test and if there were intergroup differences the Dunn test was applied. The level of significance was set at $\mathrm{P}<0.05$.

\section{Results}

A total of 63 animals were initially enrolled in the study. Nine animals died during the surgical procedure (a mortality rate of $14 \%$ ) and a total of 54 animals completed the protocol: 11 rats in $\mathrm{G} 1 ; 8$ rats in G2; 9 rats in G3; 10 rats in $\mathrm{G} 4 ; 8$ rats in $\mathrm{G} 5$, and 8 rats in $\mathrm{G} 6$. Mean body weight was smaller in groups receiving the normocholesterolemic diet than in groups receiving the hypercholesterolemic diet both at baseline and after 30 days of AMI (Table 2). Similarly, end-diastolic left ventricular diameters and volumes were smaller in animals who received the normocholesterolemic diet than in those who received the hypercholesterolemic diet. No differences were observed in left ventricular mass corrected by body weight 


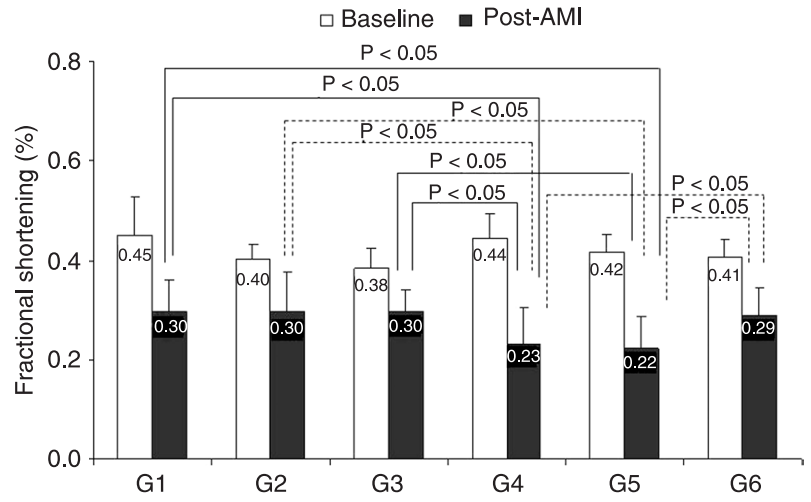

Figure 2. Fractional shortening of left ventricle. See Figure 1 for protocol. There was significant decrease in fractional shortening from baseline to the post-acute myocardial infarction condition in all groups. The variables measured in only one condition were evaluated by analysis of variance (ANOVA) with a classification factor, when significant. The Tukey test was applied to determine differences between groups. AMI = acute myocardial infarction.

and, after animals sacrifice, there was no difference in heart weight between groups. When compared to baseline, there was a significant increase in left ventricular diastolic diameter $(P<0.05)$ and left ventricular volume $(P<0.05)$ in all groups.

Figure 2 illustrates the fractional shortening in the 6 groups at baseline and 30 days after AMI. Fractional shortening was normal at baseline and decreased significantly after AMI ( $P$ $<0.05$ in all groups). After AMI, fractional shortening was lower in groups 4 (hypercholesterolemic diet without rosuvastatin) and 5 (hypercholesterolemic diet with rosuvastatin after AMI) than in the other groups. No significant difference was observed in fractional shortening between group 6 (hypercholesterolemic diet with rosuvastatin before and after $\mathrm{AMI}$ ) and groups receiving the normocholesterolemic diet.

\section{Infarct size}

Histological analysis revealed that the percentage of infarct area was significantly lower in G3 (normocholesterolemic diet with rosuvastatin 30 days before and after AMI) than in G4 (hypercholesterolemic diet without rosuvastatin), as demonstrated in Figure 3. No significant differences were observed in infarct area among the other groups.

\section{Biochemical analysis}

IL-6, MMP-2, TNF- $\alpha$, and ADMA levels before and after LAD occlusion are presented in Table 3. At baseline, no difference was observed in IL-6 or TNF- $\alpha$ between groups. After LAD occlusion, IL-6 levels decreased in G1 compared to $G 2(P<0.05)$, whereas no difference was observed in the other groups.

At baseline, MMP-2 levels were higher in $\mathrm{G} 1$ than in G3 and G5 (P < 0.05). MMP-2 levels were reduced after LAD occlusion in $G 5$ compared to $G 2(P<0.05)$ and did

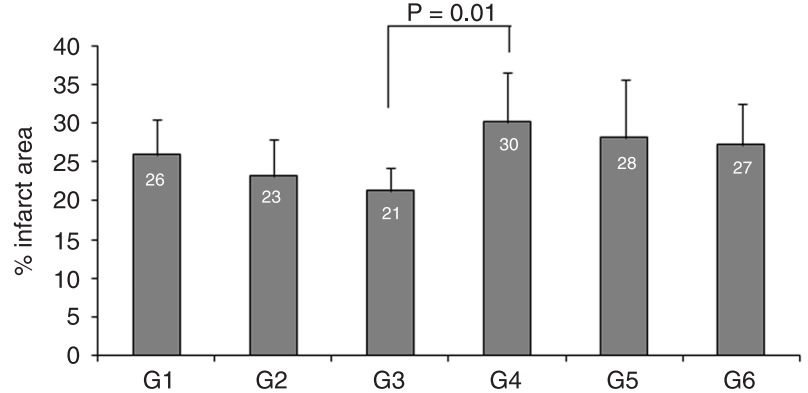

Figure 3. Percent infarct area determined by histological analysis. See Figure 1 for protocol. The data were compared between all groups. The variables measured in only one condition were evaluated by analysis of variance (ANOVA) with a classification factor, when significant. The Tukey test was applied to determine differences between groups.

Table 3. Biochemical data at baseline and after left anterior descending (LAD) coronary artery occlusion.

\begin{tabular}{cclll}
\hline & $\begin{array}{c}\mathrm{IL}-6 \\
(\mathrm{pg} / \mathrm{mL})\end{array}$ & $\begin{array}{c}\text { MMP-2 } \\
(\mathrm{pg} / \mathrm{mL})\end{array}$ & $\begin{array}{c}\text { TNF- } \alpha \\
(\mathrm{pg} / \mathrm{mL})\end{array}$ & $\begin{array}{c}\text { ADMA } \\
(\mu \mathrm{M})\end{array}$ \\
\hline $\begin{array}{c}\text { Baseline } \\
\text { G1 }\end{array}$ & 47.1 & $301.1^{*}$ & 0.00 & $1.21^{\#}$ \\
G2 & 43.9 & 268.7 & 0.07 & 1.23 \\
G3 & 28.6 & $136.2^{*}$ & 0.06 & $0.73^{\#}$ \\
G4 & 11.7 & 162.4 & 0.36 & 0.93 \\
G5 & 11.1 & $147.2^{*}$ & 0.08 & $0.77^{\#}$ \\
G6 & 53.4 & 169.5 & 0.08 & 0.81 \\
30 days after AMI & & & & \\
G1 & $6.4^{*}$ & 188.8 & $0.36^{+}$ & 0.85 \\
G2 & $84.1^{*}$ & $186.5^{\#}$ & 1.71 & 0.84 \\
G3 & 26.9 & 153.2 & 0.00 & $0.72^{\Delta}$ \\
G4 & 39.1 & 122.8 & 8.63 & 0.90 \\
G5 & 66.1 & $103.0^{\#}$ & $22.1^{+}$ & 0.85 \\
G6 & 23.2 & 132.5 & 19.2 & $1.01^{\Delta}$ \\
\hline
\end{tabular}

Data are reported as median. See Figure 1 for protocol. IL-6 = interleukin 6; MMP-2 = metalloproteinase 2; TNF- $\alpha=$ tumor necrosis factor alpha; ADMA = asymmetric dimethylarginine. Levels were compared between pre- and post-infarction conditions. Baseline: * $\mathrm{P}<0.05 \mathrm{G} 3$, G5 compared to G1 (ANOVA). ${ }^{\#} \mathrm{P}<0.05$ G3, G5 compared to G1 (ANOVA). 30 days after AMI: ${ }^{*} \mathrm{P}<0.05$ G1 compared to G2 (ANOVA). \#P < 0.05 G5 compared to G2 (ANOVA). ${ }^{+} P<0.05 \mathrm{G} 1$ compared to $\mathrm{G} 5$ (ANOVA). ${ }^{\Delta} \mathrm{P}<0.05 \mathrm{G} 3$ compared to G6 (ANOVA).

not change in the other groups $(P=N S)$.

After LAD occlusion, TNF- $\alpha$ levels were reduced in $\mathrm{G} 1$ compared to $G 5(P<0.05)$, whereas no difference was observed in the other groups. Before LAD occlusion, ADMA levels were higher in $\mathrm{G} 1$ than in $\mathrm{G} 3$ and $\mathrm{G} 5$ and were reduced after LAD occlusion in $G 3$ compared to $G 6(P<0.05)$. 


\section{Discussion}

In this study, we demonstrated that a normocholesterolemic diet associated with rosuvastatin 30 days before and 30 days after AMI resulted in a decreased percentage of myocardial necrosis area compared to a hypercholesterolemic diet. Interestingly, the hypercholesterolemic diet resulted in depressed left ventricular systolic function after AMI and this effect was attenuated by treatment with rosuvastatin 30 days before and after AMI (but not by treatment after AMI alone). We believe that these data would be of important benefit for the treatment of infarcts if these achievements were confirmed in human models. We know that, before extrapolating these data to clinical practice, it would be important to confirm them in experiments of longer duration to evaluate ventricular function and remodeling, and also in other animal models such as pig or dogs whose coronary system is closer to the human one.

According to Davignon and Laaksonen (21), statins have several effects related to the reduction of LDL-C levels. However, this class of drugs has other metabolic manifestations such as pleiotrophic effects that are independent of cholesterol reduction. The fundamental question that remains is whether these effects promote additional cardioprotection than the known LDL-C-reducing action. Rosenson and Tangney (22) have suggested 4 non-lipidic mechanisms that may contribute to the beneficial effects on clinical events. These effects include modification of endothelial function, inflammatory response, plaque stabilization, and thrombus formation.

Findings showing that pharmacological inhibition of MMP attenuates the dilation of the left ventricle in infarcted rats have led to the conclusion that the mechanisms of MMP inhibitors may have therapeutic potential in patients at risk of developing heart failure after AMI (23). In a retrospective study, Lagrand et al. (24) concluded that C-reactive protein is a marker of risk factors for cardiovascular disease based on still unknown molecular mechanisms. In a clinical study, Strandberg et al. (25) observed a significant decrease in C-reactive protein levels using atorvastatin and simvastatin for the treatment of dyslipidemia, as also observed in the CARE study (26).

Inflammatory cytokines secreted by macrophages and T lymphocytes may modify endothelial function and influence the proliferation of smooth muscle cells, degradation of collagen, and thrombosis (27). In an experimental study on dyslipidemic animals, Scalia et al. (28) observed an increased expression of P-selectin activity, the intercellular adhesion molecule (ICAM-1) and the adhesion molecule of vascular cell (VCAM-1) in the interaction of leukocytes with the endothelium.

In clinical situations, such as hypercholesterolemia, the bioavailability of nitric oxide is reduced, among other factors, by increased levels of an endogenous competitive inhibitor of eNOS, ADMA, which assumes the function of regulating the formation of nitric oxide that preserves the important vascular tone (29). Serum ADMA levels were significantly higher in hypercholesterolemic individuals compared to normocholesterolemic individuals and the $\mathrm{L}$-arginine/ADMA ratio was decreased in these patients (30).

Zoccali et al. (31) also pointed out the multifaceted origin of atherosclerosis, emphasizing that endothelial dysfunction, the initial insult, is characterized by increased adhesiveness of the endothelium to leukocytes and platelets and the synthesis of molecules with vessel activity, cytokines and pro-coagulation factors. Cytokines, low molecular weight proteins involved in inflammation and in the responses of the immune system, are considered to be independent risk factors for coronary artery and cerebrovascular diseases (32). Among them are TNF- $\alpha$, IL- 6 and the inhibitor of plasminogen activator factor 1 (33).

In our study, we did not observe reduction of TNF-a, IL-6 or ADMA levels in the groups treated with rosuvastatin. No correlation was observed between biochemical data and infarcted area size, showing no direct correlation between the biochemical expressions and hemodynamic repercussion of the infarct. However, we did demonstrate a reduction in infarcted area size in normocholesterolemic animals treated with rosuvastatin before AMI and during the healing period. These data indicate the need for clinical studies to confirm whether the use of a statin by normocholesterolemic patients can reduce infarct size and improve ventricular function.

\section{Limitations}

Infarct size using this model is not uniform, mainly because of the inability to occlude the coronary artery exactly at the same point in all animals, as well as because of eventual anatomical variations. Since infarct area risk was not determined in our study, infarct size was reported as the percentage of total left ventricular area.

Certainly there would be differences between coronary atherosclerosis and our model of infarction in rats without previous coronary artery disease, but the final mechanisms would be similar because acute vessel occlusion was provoked. We observed an increase in cholesterol levels in the groups on a hypercholesterolemic diet, but there were no significant differences in endothelial dysfunction evaluated by ADMA or in inflammatory biomarkers. The only difference observed was in MMP-2 levels, which were lower in the hypercholesterolemic group treated with rosuvastatin (G5), demonstrating a possible role of rosuvastatin in protecting against myocardial damage by some mechanism that causes less MMP-2 expression. This may lead to mural realignment of myocyte clusters and/or of individual myocytes within the left ventricular wall, causing thinning and dilation of the ventricular wall and consequent heart failure. Moreover, the pathological degradation of collagen after various myocardial episodes of ischemia 
was associated with increased inflammatory activity in tissues and an increase of angiotensin II and aldosterone, which also provide the remodeling. Thus, the present data demonstrating a reduction in MMP-2 expression in the hypercholesterolemic group could be important for the evaluation of the development of ventricular remodeling.

\section{References}

1. Pekkanen J, Linn S, Heiss G, Suchindran CM, Leon A, Rifkind BM, et al. Ten-year mortality from cardiovascular disease in relation to cholesterol level among men with and without preexisting cardiovascular disease. N Engl J Med 1990; 322: 1700-1707.

2. Chagas AC, Da-Luz PL, Pileggi F. Infarct-sparing effect of propranolol in an occlusion-reperfusion dog model. Braz $J$ Med Biol Res 1989; 22: 1337-1345.

3. Da-Luz PL, Silveira MC, Chagas AC, Pileggi F. Myocardial protection by verapamil and reperfusion following coronary occlusion. Braz J Med Biol Res 1990; 23: 317-324.

4. The Thrombolysis in Myocardial Infarction (TIMI) trial. Phase I findings. TIMI Study Group. N Engl J Med 1985; 312: 932936.

5. Effectiveness of intravenous thrombolytic treatment in acute myocardial infarction. Gruppo Italiano per lo Studio della Streptochinasi nell'Infarto Miocardico (GISSI). Lancet 1986; 1: 397-402.

6. Kloner RA, Ellis SG, Lange R, Braunwald E. Studies of experimental coronary artery reperfusion. Effects on infarct size, myocardial function, biochemistry, ultrastructure and microvascular damage. Circulation 1983; 68: I-8-I-15.

7. Diamond GA, Forrester JS, deLuz PL, Wyatt HL, Swan HJ. Post-extrasystolic potentiation of ischemic myocardium by atrial stimulation. Am Heart J 1978; 95: 204-209.

8. Brown MS, Dana SE, Goldstein JL. Regulation of 3-hydroxy3-methylglutaryl coenzyme A reductase activity in human fibroblasts by lipoproteins. Proc Natl Acad Sci U S A 1973; 70: 2162-2166.

9. Aguilar-Salinas CA, Barrett H, Schonfeld G. Metabolic modes of action of the statins in the hyperlipoproteinemias. Atherosclerosis 1998; 141: 203-207.

10. Nissen SE, Nicholls SJ, Sipahi I, Libby P, Raichlen JS, Ballantyne $\mathrm{CM}$, et al. Effect of very high-intensity statin therapy on regression of coronary atherosclerosis: the ASTEROID trial. JAMA 2006; 295: 1556-1565.

11. Kjekshus J, Apetrei E, Barrios V, Bohm M, Cleland JG, Cornel $\mathrm{JH}$, et al. Rosuvastatin in older patients with systolic heart failure. N Engl J Med 2007; 357: 2248-2261.

12. Ridker PM, Danielson E, Fonseca FA, Genest J, Gotto AM $\mathrm{Jr}$, Kastelein JJ, et al. Rosuvastatin to prevent vascular events in men and women with elevated C-reactive protein. N Engl J Med 2008; 359: 2195-2207.

13. Terp K, Koudahl V, Veien M, Kim WY, Andersen HR, Baandrup $U$, et al. Functional remodelling and left ventricular dysfunction after repeated ischaemic episodes. A chronic experimental porcine model. Scand Cardiovasc J 1999; 33: 265-273.

14. Mebazaa A, Wetzel R, Cherian M, Abraham M. Comparison between endocardial and great vessel endothelial cells: mor-
A hypercholesterolemic diet in rats resulted in reduced cardiac function after AMI, which was reversed by rosuvastatin when started before AMI. A normocholesterolemic diet associated with rosuvastatin before and after AMI prevented myocardial necrosis compared to the hypercholesterolemic condition.

phology, growth, and prostaglandin release. Am J Physiol 1995; 268: H250-H259.

15. Tyagi SC, Matsubara L, Weber KT. Direct extraction and estimation of collagenase(s) activity by zymography in microquantities of rat myocardium and uterus. Clin Biochem 1993; 26: 191-198.

16. Scheuren N, Jacobs M, Ertl G, Schorb W. Cyclooxygenase-2 in myocardium stimulation by angiotensin-II in cultured cardiac fibroblasts and role at acute myocardial infarction. $J \mathrm{Mol}$ Cell Cardiol 2002; 34: 29-37.

17. Thorgeirsson UP, Lindsay CK, Cottam DW, Gomez DE. Tumor invasion, proteolysis, and angiogenesis. J Neurooncol 1994; 18: 89-103.

18. Position of the American Heart Association on research animal use. Circulation 1985; 71: 849A-850A.

19. Joris I, Zand T, Nunnari JJ, Krolikowski FJ, Majno G. Studies on the pathogenesis of atherosclerosis. I. Adhesion and emigration of mononuclear cells in the aorta of hypercholesterolemic rats. Am J Pathol 1983; 113: 341-358.

20. Galvão TFG, Matos K, Rolim NPL, Brum PC, Negrão CE, Da Luz PL, et al. Low-to-moderate intensity exercise training reduces myocardial infarct size in rats. Eur Heart J 2006; 27: 250.

21. Davignon J, Laaksonen R. Low-density lipoprotein-independent effects of statins. Curr Opin Lipidol 1999; 10: 543559.

22. Rosenson RS, Tangney CC. Antiatherothrombotic properties of statins: implications for cardiovascular event reduction. JAMA 1998; 279: 1643-1650.

23. Peterson JT, Li H, Dillon L, Bryant JW. Evolution of matrix metalloprotease and tissue inhibitor expression during heart failure progression in the infarcted rat. Cardiovasc Res 2000; 46: 307-315.

24. Lagrand WK, Visser CA, Hermens WT, Niessen HW, Verheugt FW, Wolbink GJ, et al. C-reactive protein as a cardiovascular risk factor: more than an epiphenomenon? Circulation 1999; 100: 96-102.

25. Strandberg TE, Vanhanen H, Tikkanen MJ. Effect of statins on C-reactive protein in patients with coronary artery disease. Lancet 1999; 353: 118-119.

26. Sacks FM, Pfeffer MA, Moye LA, Rouleau JL, Rutherford $J D$, Cole TG, et al. The effect of pravastatin on coronary events after myocardial infarction in patients with average cholesterol levels. Cholesterol and Recurrent Events Trial investigators. N Engl J Med 1996; 335: 1001-1009.

27. Koh KK. Effects of statins on vascular wall: vasomotor function, inflammation, and plaque stability. Cardiovasc Res 2000; 47: 648-657.

28. Scalia R, Appel JZ III, Lefer AM. Leukocyte-endothelium interaction during the early stages of hypercholesterolemia 
in the rabbit: role of P-selectin, ICAM-1, and VCAM-1. Arterioscler Thromb Vasc Biol 1998; 18: 1093-1100.

29. Boger RH. Asymmetric dimethylarginine (ADMA): a novel risk marker in cardiovascular medicine and beyond. Ann Med 2006; 38: 126-136.

30. Boger RH, Bode-Boger SM, Szuba A, Tsao PS, Chan JR, Tangphao $\mathrm{O}$, et al. Asymmetric dimethylarginine (ADMA): a novel risk factor for endothelial dysfunction: its role in hypercholesterolemia. Circulation 1998; 98: 1842-1847.
31. Zoccali C, Mallamaci F, Tripepi G. Inflammation and atherosclerosis in end-stage renal disease. Blood Purif 2003; 21: 29-36.

32. Yang RZ, Lee MJ, Hu H, Pollin TI, Ryan AS, Nicklas BJ, et al. Acute-phase serum amyloid $A$ : an inflammatory adipokine and potential link between obesity and its metabolic complications. PLoS Med 2006; 3: e287.

33. de Carvalho MH, Colaco AL, Fortes ZB. [Cytokines, endothelial dysfunction, and insulin resistance]. Arq Bras Endocrinol Metabol 2006; 50: 304-312. 